\title{
МЕДИКАМЕНТОЗНАЯ ПРОФИЛАКТИКА САХАРНОГО ДИАБЕТА 2-ГО ТИПА У ПАЦИЕНТОВ С РАННИМИ НАРУШЕНИЯМИ УГЛЕВОДНОГО ОБМЕНА: ЭФФЕКТИВНОСТЬ И КЛИНИЧЕСКИЕ ИСХОДЫ ПРИ ДЛИТЕЛЬНОМ НАБЛЮДЕНИИ
}

\author{
В. В. Боева ${ }^{1 凶}$, А. Н. Завьялов
}

1 Федеральный клинический центр высоких медицинских технологий Федерального медико-биологического агентства России, Химки, Московская область, Россия

2 Российский национальный исследовательский медицинский университет имени Н. И. Пирогова Минздрава России, Москва, Россия

\begin{abstract}
Актуальность профилактики сахарного диабета (СД) 2-го типа у пациентов с предиабетом увеличивается из-за неуклонного распространения заболевания. Целью работы было оценить эффективность медикаментозной профилактики в замедлении темпов конверсии впервые выявленных нарушенной толерантности к глюкозе и нарушенной гликемии натощак (НТГ/НГН) в СД 2-го типа. Участникам исследования (1136 человек), считавшимся здоровыми после диспансеризации, повторно провели скрининг СД 2-го типа с соблюдением правил преаналитической подготовки образцов крови. Пациентам с впервые выявленными НТГ/НГН была назначена терапия метформином и/или акарбозой, частоту конверсии НТГ/НГН в СД 2-го типа оценивали через 3 и 10 лет наблюдения. У 18,5\% ( $n=210)$ обследованных выявили различные категории нарушения углеводного обмена: СД 2-го типа - у 5,0\%, НТГ — у 5,5\%, НГН - у 8,0\%. Пациентам с впервые выявленным СД 2-го типа была назначена сахароснижающая терапия, они были исключены из последующего наблюдения. Пациентам с НТГ/НГН $(n=151)$ рекомендовали изменение образа жизни и назначили терапию метсормином $(77 \%)$ или метформином и акарбозой (23\%). Частота конверсии СД 2-го типа в течение 3 лет активного наблюдения составила 6,8\% на фоне монотерапии метформином и 11,4\% - на фоне комбинированной терапии метформином и акарбозой. По окончании периода активного наблюдения большинство пациентов ( $(=85)$ самостоятельно прекратили терапию. Частота конверсии НТГ/НГН в СД 2-го типа через 10 лет после окончания активного наблюдения в группе без медикаментозной профилактики составила 38,8\% и 0\% - в группе принимающих метформин ( $<<0,01)$. Показано, что длительное применение метформина предупредило развитие СД 2-го типа в отдаленном периоде у 83,3\% (p < 0,05).
\end{abstract}

Ключевые слова: сахарный диабет 2-го типа, нарушенная гликемия натощак, нарушенная толерантность к глюкозе, скрининг, метформин, акарбоза, профилактика, глюкоза венозной плазмы натощак

Вклад авторов: В. В. Боева - планирование исследования, анализ литературы, сбор, анализ и интерпретация данных, подготовка черновика рукописи; А. Н. Завьялов - анализ литературы, анализ и интерпретация данных, подготовка черновика рукописи.

Соблюдение этических стандартов: исследование одобрено этическим комитетом РНИМУ имени Н. И. Пирогова (протокол № 176 от 25 июня 2018 г.). Все пациенты подписали добровольное информированное согласие на участие в исследовании.

$凶$ Для корреспонденции: Валентина Владимировна Боева

ул. Ивановская, д. 15А, г. Химки, Московская область, 141435; boevaWV@yandex.ru

Статья получена: 09.01.2020 Статья принята к печати: 08.02.2020 Опубликована онлайн: 06.03.2020

DOI: $10.24075 /$ vrgmu.2020.014

\section{PREVENTIVE PHARMACOTHERAPY OF TYPE 2 DIABETES MELLITUS IN PATIENTS WITH EARLY CARBOHYDRATE METABOLISM DISORDERS: LONG-TERM EFFICACY AND CLINICAL OUTCOMES}

Boeva $\mathrm{W}^{1} \square$, Zavyalov $\mathrm{AN}^{2}$

${ }^{1}$ Federal Clinical Center of High Medical Technologies, FMBA, Khimki, Moscow region, Russia

2 Pirogov Russian National Research Medical University, Moscow, Russia

Prevention of type 2 diabetes mellitus (T2DM) in prediabetic patients is a pressing concern due to its increasing prevalence. The aim of this study was to evaluate the efficacy of preventive pharmacotherapy in delaying progression of incident impaired glucose tolerance (IGT) and impaired fasting glycemia (IFG) to T2DM. The participants of the study (1,136 subjects) found healthy by a regular annual checkup underwent repeat screening for T2DM. Blood samples were processed following the guidelines for good preanalytical sample preparation. Patents with incident IGT/IFG were prescribed medication therapy with metformin or/and acarbose. The rate of IGT/IFG conversion to T2DM was evaluated in years 3 and 10 of observation. Carbohydrate metabolism disorders were detected in $18.5 \%$ ( $n=210$ ) of the re-screened patients: 5.0\% had T2DM, 5.5\% had IGT, 8.0\% had IFG. Patients with incident T2DM were prescribed blood sugar lowering therapy and they were excluded from further analysis. Patients with IGT/IFG $(n=151)$ were given recommendations on lifestyle modification and prescribed metformin ( $77 \%)$ or a combination of metformin and acarbose (23\%). Three years after the start of observation, the rate of conversion to T2DM was $6.8 \%$ in patients undergoing monotherapy with metformin and $11.4 \%$ in patients undergoing combination therapy with metformin and acarbose. After the active follow-up phase was over, the majority of the patients $(n=85)$ decided to discontinue preventive therapy without consulting their physicians. Ten years after the active follow-up phase, the rate of NGT/IFG conversion to T2DM was 38.8\% in patients who had discontinued their treatment and $0 \%$ in patients still taking metformin ( $p<0.01$ ). Long-term therapy with metformin prevented progression to T2DM in the long run in $83.3 \%(p<0.05)$.

Keywords: type 2 diabetes mellitus, impaired fasting glucose, impaired glucose tolerance, screening, metformin, acarbose, prevention, fasting plasma glucose

Author contribution: Boeva W planned the study; analyzed the literature; collected, analyzed and interpreted study results; wrote the manuscript; Zavyalov AN analyzed the literature; analyzed and interpreted study results; wrote the manuscript.

Compliance with ethical standards: the study was approved by the Ethics Committee of Pirogov Russian National Research Medical University (Protocol № 176 dated June 25, 2018). Informed consent was obtained from all study participants.

$\triangle$ Correspondence should be addressed: Valentina V. Boeva

Ivanovskaya, 15A, Khimki, Moscow region, 141435; boevaW@yandex.ru

Received: 09.01.2020 Accepted: 08.02.2020 Published online: 06.03.2020

DOI: 10.24075/brsmu.2020.014 
Сахарный диабет (СД) 2-го типа - важнейшее социально значимое заболевание, характеризующееся высокой и постоянно растущей распространенностью, повышением риска инвалидизации и ранней смертности. В России на январь 2018 г. зарегистрировано 4,1 млн больных СД 2-го типа [1, 2]. Однако результаты контрольноэпидемиологических исследований свидетельствуют, что реальное число больных в России может составлять не менее 8 млн человек. Экстраполяция результатов исследования NATION на всех жителей РФ позволяет предполагать, что около 20,7 млн человек имеют недиагностированный предиабет [3] и, следовательно, не получают необходимые рекомендации и терапию.

В настоящее время пациенты с нарушенной толерантностью к глюкозе и нарушенной гликемией натощак (НТГ/НГН) не подлежат обязательному учету, их диспансерное наблюдение не ведется, в связи с чем реальная распространенность предиабета изучена недостаточно.

Главная причина несвоевременной диагностики СД 2-го типа и ранних стадий нарушений углеводного обмена нарушение правил преаналитической подготовки образцов крови, направленных на подавление гликолиза. Именно гликолиз в форменных элементах крови, находящейся в пробирке после ее забора у пациента, приводит к снижению уровня глюкозы в образце и занижает конечный результат. Таким образом, у части лиц с нарушениями углеводного обмена (в нашем исследовании - 18,5\%) во время скрининга выявляют нормальные показатели глюкозы, и данная группа пациентов не попадает под наблюдение врачей.

Врачи первичного звена не всегда придают должное значение выявленным НГН/НТГ. По этой причине советы по изменению образа жизни нередко носят формальный характер, медикаментозную профилактику не назначают, а приверженность пациентов к рекомендациям оказывается низкой. $\mathrm{K}$ настоящему времени эффективность медикаментозного лечения лиц с предиабетом подтверждена в многочисленных исследованиях [4-7] и метаанализах [8].
$\mathrm{Ha}$ сегодняшний день необходимы активное выявление ранних форм нарушений углеводного обмена и их коррекция. Целью работы было в условиях реальной клинической практики оценить эффективность медикаментозной профилактики в замедлении темпов конверсии впервые выявленных НТГ/НГН в СД 2-го типа в отдаленном периоде.

\section{ПАЦИЕНТЫ И МЕТОДЫ}

\section{Участники исследования}

В исследовании приняли участие 1136 взрослых пациентов, проживающих в Тамбовской области, обратившихся в Тамбовскую ЦРБ в рамках программы дополнительной диспансеризации в 2007 г. Все пациенты считались здоровыми после проведенной плановой диспансеризации. Критерии включения пациентов в исследование: отсутствие нарушений углеводного обмена в анамнезе; отсутствие сахароснижающей терапии в анамнезе. Критерии исключения: выявление по результатам скрининга СД 1-го или 2-го типов.

\section{Этапы исследования}

1. Скрининг на наличие нарушений углеводного обмена у лиц, которых считали здоровыми после проведенной плановой диспансеризации, медикаментозная просилактика СД 2-го типа при наличии показаний, оценка частоты конверсии НТГ/НГН в СД 2-го типа и нормогликемии в течение трех лет активного наблюдения. 2. Оценка долгосрочных исходов: частоты конверсии НТГ/НГН в СД 2-го типа и нормогликемии через 10 лет после окончания периода активного наблюдения (рис. 1).

Первый этап следует рассматривать как нерандомизированное сплошное проспективное интервенционное исследование, второй этап нерандомизированное ретроспективное наблюдательное контролируемое исследование.

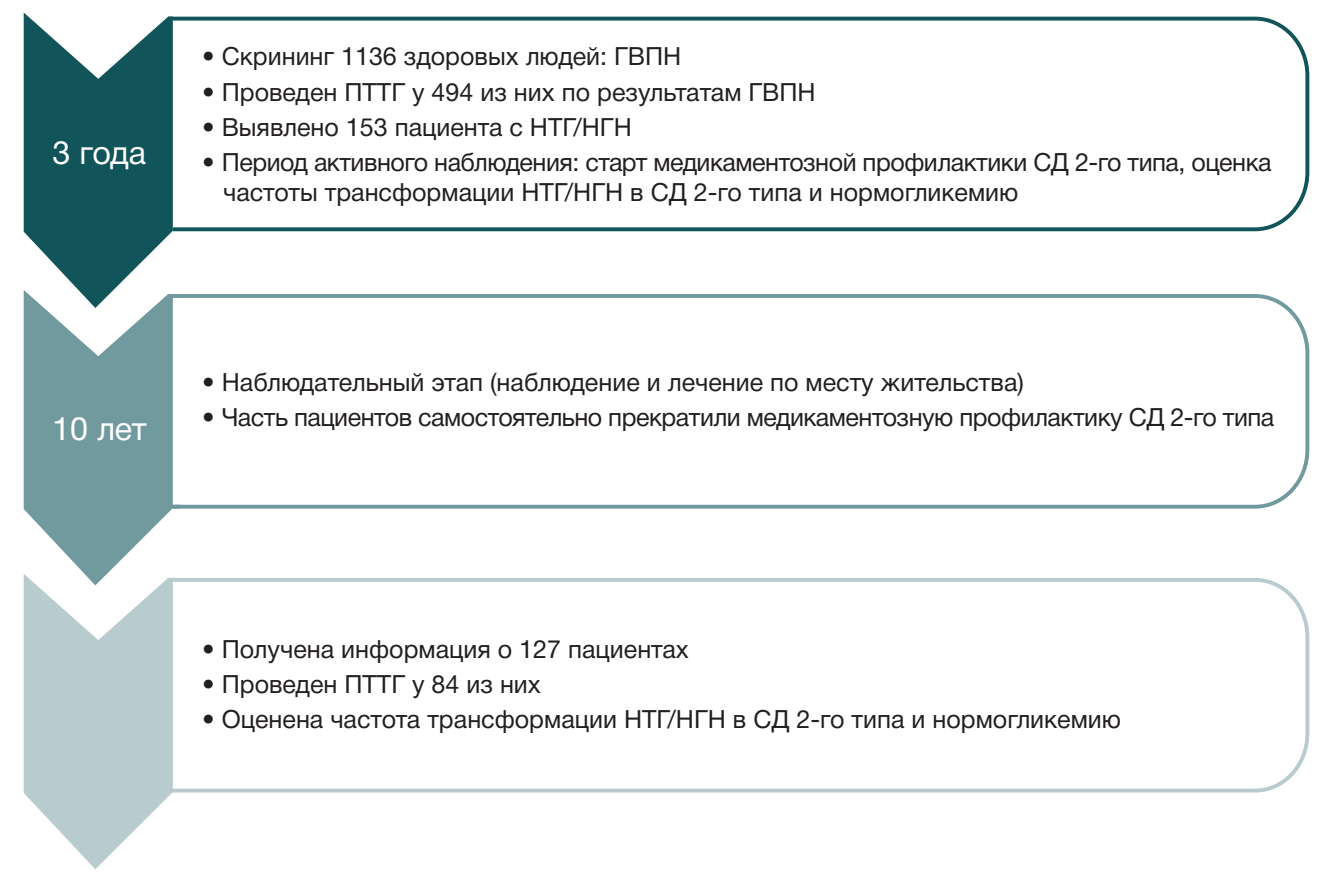

Рис. 1. Схема проведения исследования. ГВПН - глюкоза венозной плазмы натощак, ПТтГ — пероральный тест толерантности к глюкозе (после нагрузки, включающей прием 75 г глюкозы) 


\section{Продолжительность исследования}

Продолжительность периода активного наблюдения, в том числе медикаментозной профилактики СД 2-го типа составила 3 года. Часть пациентов продолжила медикаментозную профилактику (максимум 13 лет). Долговременная эффективность терапии была оценена через 10 лет после окончания активного наблюдения.

\section{Описание медицинского вмешательства}

Диагностику категорий нарушения углеводного обмена проводили на основании результатов скрининга, включая проведение перорального теста толерантности к глюкозе (ПТТГ). При выявлении НТГ/НГН давали рекомендации по модификации образа жизни и назначали медикаментозную терапию. Через 10 лет после окончания периода активного наблюдения все доступные для контакта пациенты $(n=115)$ были обследованы повторно. В качестве основного показателя исследования оценивали частоту конверсии НТГ/НГН в СД 2-го типа и нормогликемию в течение периода активного наблюдения и медикаментозной профилактики (3 года) и через 10 лет после его окончания.

\section{Анализ в подгруппах}

На этапе скрининга проводили стратификацию по наличию метаболического синдрома и факторов риска СД 2-го типа.

Исходы по состоянию углеводного обмена через 10 лет стратисицированы в зависимости от наличия/отсутствия долговременной медикаментозной профилактики СД 2-го типа. Соответственно в отдаленном периоде мы подразделили доступных для анализа пациентов $(n=115)$ на две подгруппы: пациенты первой подгруппы самостоятельно прекратили медикаментозную профилактику СД 2-го типа ( $n=85)$, пациенты второй подгруппы принимали метформин в течение всего срока наблюдения $(n=30)$.

\section{Методы регистрации исходов}

Использовали критерии нарушений углеводного обмена, рекомендованные Всемирной организацией здравоохранения (ВОЗ) в 1999 г. Для оценки состояния углеводного обмена применяли общепринятую двухточечную двухчасовую методику ПТТГ с 75 г глюкозы. Во всех случаях концентрацию глюкозы определяли в лаборатории Тамбовской ЦРБ, забор крови проводили в пробирки с фторидом натрия, пробоподготовку — по единой преаналитической методике.

\section{Статистический анализ}

Результаты исследования оценивали с помощью программы Statistica 6.1 (ТІВCO; США). Использовали методы непараметрической статистики, метод $\chi^{2}$ Пирсона, точный критерий Фишера, расчет поправки Йетса таблиц сопряженности; достоверность различий оценивали как статистически значимую при $p<0,05$.

\section{РЕЗУЛЬТАТЫ ИССЛЕДОВАНИЯ}

Исходно скрининг проведен у 1136 человек, считавших себя абсолютно здоровыми. У 73 (6,4\%) человек, у которых уровень глюкозы венозной плазмы натощак (ГВПН) составил $\geq$ 7,0 ммоль/л, было проведено повторное исследование ГВПН, и у 37 пациентов был подтвержден диагноз СД 2-го типа. Этим больным была назначена терапия и из дальнейшего наблюдения они были исключены. У остальных 36 пациентов гликемия оказалась в пределах 6,1-6,9 ммоль/л. Этим лицам и еще 181 из 1136 обследованных, у которых при скрининге выявили гликемию в тех же пределах, был проведен ПТТГ.

У 882 из 1136 (77,6\%) ГВПН оказалась ниже 6,1 ммоль/л, что в соответствии с действующими критериями ВОЗ является нормой и не требует диагностики СД в ближайшее время. Однако IDF (2005 г.) рекомендует проводить ПТТГ
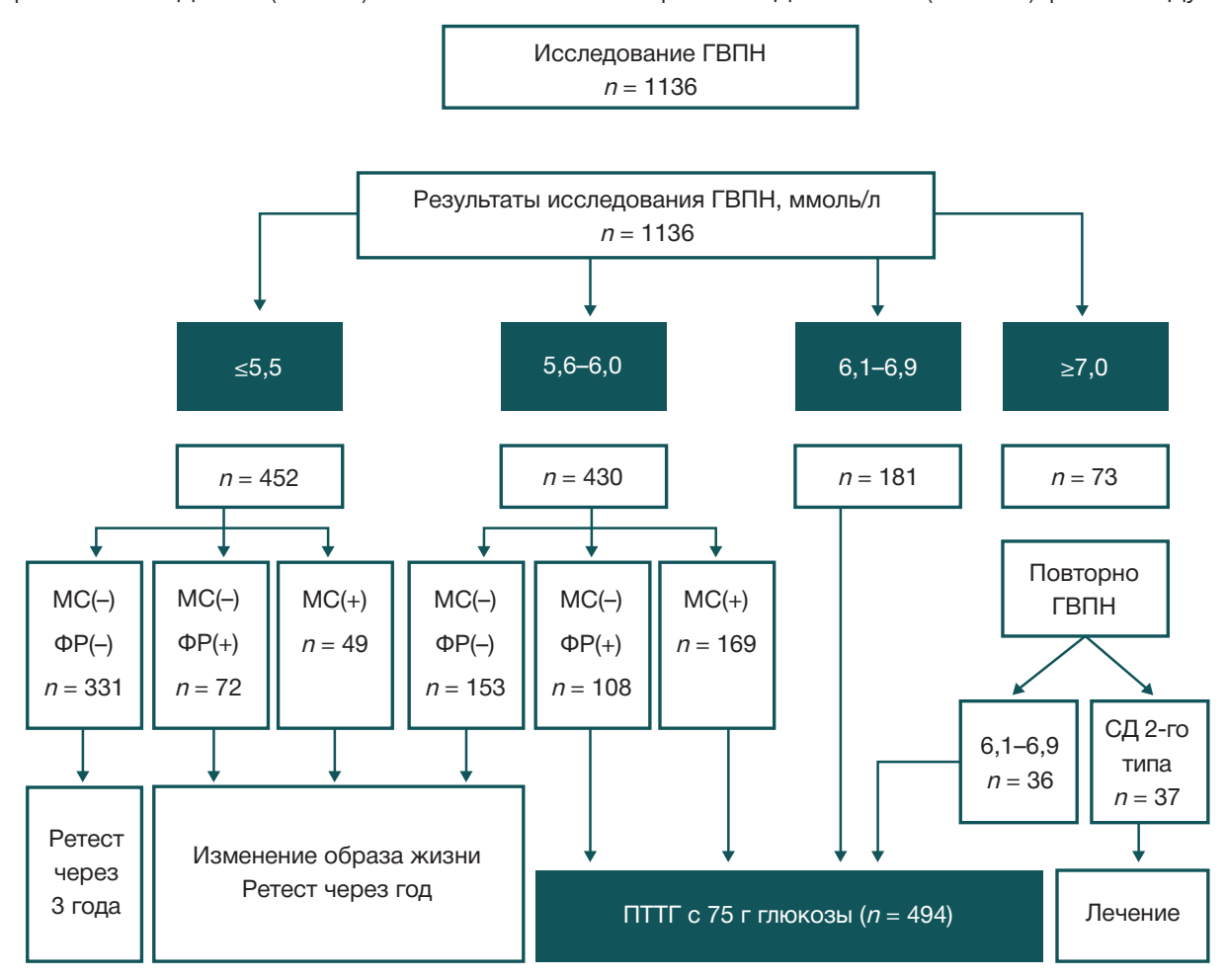

Рис. 2. Распределение больных по результатам скрининга для дальнейшей диагностики СД 2-го типа. ГВПН - глюкоза венозной плазмы натощак; МС метаболический синдром; ПтТГ — пероральный тест толерантности к глюкозе с 75 г глюкозы; ФР — факторы риска 
всем лицам с метаболическим синдромом (MC), у которых гликемия натощак $\geq 5,6$ ммоль/л. Среди обследуемых нами лиц у 430 человек ГВПН оказалась в пределах 5,66,0 ммоль/л. Признаки МС были выявлены у 169 из 430 $(39,3 \%)$ человек. Им, в соответствии с рекомендациями IDF, был проведен ПТТГ. У оставшегося 261 человека полной картины МС выявлено не было, однако у 108 из них имелись факторы риска развития СД 2-го типа, в связи с чем им тоже был проведен ПТТГ (рис. 2). Таким образом, ПТТГ был выполнен у 494 человек.

В ходе ПТТГ с 75 г глюкозы было выявлено, что у 20 человек (4,0\%) - СД 2-го типа, у 62 человек (12,6\%) нарушенная толерантность к глюкозе, у 91 человека (18,4\%) - нарушенная гликемия натощак и у 321 человека (65,0\%) - нормальная толерантность к глюкозе.

Описание исходных характеристик пациентов, включенных в исследование, опубликовано ранее [9].

Среди 153 пациентов с предиабетом у 2 (1,3\%) были выявлены противопоказания к метформину и 26 (16,9\%) отказались от приема акарбозы по финансовым причинам. Таким образом, группы обследуемых в первый год активного наблюдения составили: 90 пациентов с НГН на терапии метформином в дозе 500 мг/сут., 26 пациентов с НТГ на терапии метформином в дозе 500 мг/сут. и 35 пациентов с НТГ на комбинированном лечении метформином в дозе 500 мг/сут. и акарбозой с титрацией 50-150 мг по схеме, указанной в инструкции препарата. Повторные ПТТГ в группах предиабета и исходной нормальной толерантности к глюкозе были проведены на втором и третьем годах периода активного наблюдения. На третий год при проведении контрольного ПТТГ СД 2-го типа был выявлен: в группе нормы у четырех человек $(1,4 \%)$, в группе НГН на терапии меторормином у одного человека (1,1\%), в группе НТГ на терапии метформином у двух человек (8,7\%), в группе НТГ на терапии меторормином и акарбозой у двух человек (6,1\%). По окончании третьего года наблюдения количество лиц с НТГ/НГН составляло 156 человек (рис. 3).

После окончания периода активного наблюдения у эндокринолога большинство пациентов самостоятельно прекратили терапию, несмотря на предшествующие рекомендации ее продолжать и поддерживать изменения в образе жизни.

Из 156 пациентов с НТГ/НГН в анализ отдаленных результатов медикаментозной профилактики СД 2-го типа через 10 лет было включено 115 человек. Причины исключения из анализа долгосрочных исходов остальных пациентов представлены на рис. 4.

Из 115 человек, доступных для анализа, 30 (26,1\%) принимали метформин в суточной дозе 500 мг как минимум до окончания сбора данных для настоящего исследования (2018 г.) и 85 человек самостоятельно прекратили терапию, из них 74 - практически сразу. Распределение медикаментозной профилактики по типу и продолжительности после окончания периода активного наблюдения представлено на рис. 5.

Причины низкой приверженности не были проанализированы подробно в нашем исследовании, но следует отметить, что пациенты сообщали о факторах

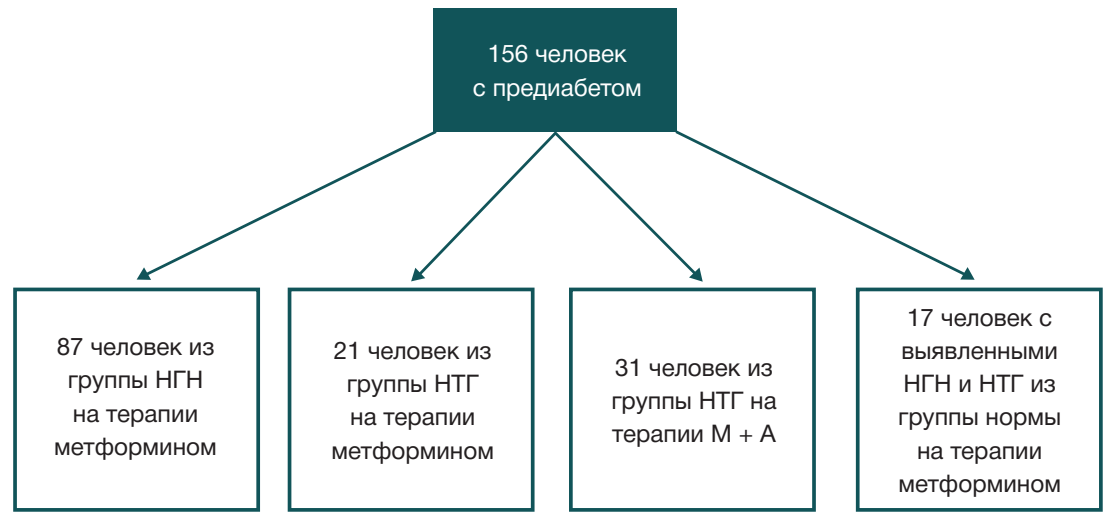

Рис. 3. Распределение больных по окончании третьего года наблюдения в зависимости от исходного состояния углеводного обмена и получаемой терапии M + A - терапия метформином и акарбозой

Пациенты с НГН/НТГ по окончании 3-го года активного наблюдения $n=156$

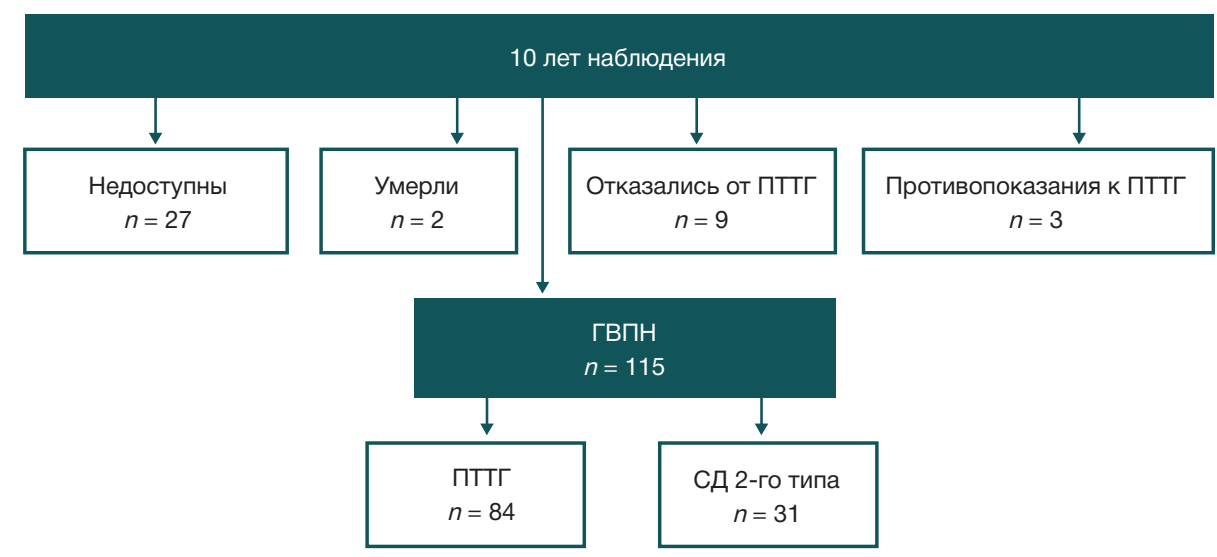

Рис. 4. Распределение больных с НТГ/НГН через 10 лет после окончания периода активного наблюдения 


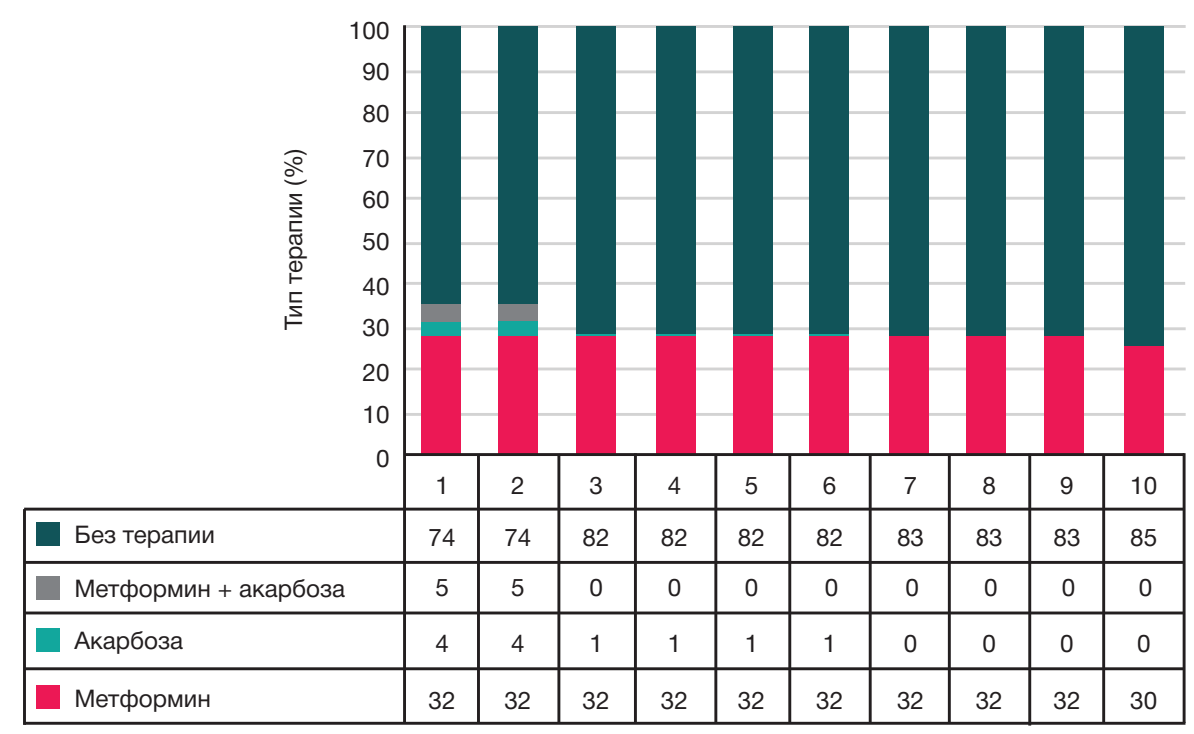

Рис. 5. Распределение медикаментозной просилактики по типу и продолжительности после окончания периода активного наблюдения (с 1-го по 10-й год)

не только медицинского, но и административного/ экономического характера. Так, отсутствие постановки на диспансерный учет в лечебно-профилактическом учреждении пациентов с предиабетом и последующий отказ им в выписке препаратов для медикаментозной профилактики чаще всего являлись причиной снижения комплаентности пациентов.

\section{Результаты медикаментозной терапии}

В период активного наблюдения из 151 человека группы предиабета у 12 (7,9\%) пациентов произошла трансформация начальных нарушений углеводного обмена в СД 2-го типа.

На фоне медикаментозной просилактики повторные ПтТГ были проведены на втором и третьем годах активного наблюдения. В группе НГН на фоне терапии метформином значимые результаты получены у пациентов с исходной ГВПН 5,6-6,0 ммоль/л, у которых нормализация углеводного обмена была достигнута в 47,8\% случаев на втором году и в 72,9\% случаев на третьем году наблюдения $\left(\chi^{2}=6,195\right.$; $p=0,013)$. Результаты медикаментозной профилактики СД 2-го типа в группе НГН в период активного наблюдения изложены нами ранее [9].

В группе НТГ пациенты, получавшие лечение метсрормином и акарбозой, продемонстрировали лучшие результаты по сравнению с монотерапией метформином: нормальная толерантность к глюкозе была зарегистрирована в большей половине случаев на третий год активного наблюдения $\left(\chi^{2}=7,222 ; p=0,007\right)$ именно на фоне комбинированной терапии.

Из 115 человек, доступных для анализа через 10 лет, $30(26,1 \%)$ принимали метформин в суточной дозе 500 мг как минимум до окончания сбора данных для настоящего исследования (2018г.), такого же длительного приема акарбозы зафиксировано не было (табл. 1).

Оценивая состояние углеводного обмена в отдаленном периоде, следует отметить, что у пациентов, находившихся на непрерывном приеме метформина, не было зарегистрировано ни одного случая развития СД 2-го типа и была достигнута более высокая частота нормализации гликемических показателей по сравнению с лицами без медикаментозной профилактики $(p<0,05)$. Через 10 лет после окончания активного наблюдения частота конверсии НТГ/НГН в СД 2-го типа составила 38,8\% в группе без медикаментозной профилактики и 0\% в группе метсормина $(p<0,01)$.

Метформин одинаково эффективен для профилактики развития СД 2-го типа вне зависимости от вида исходного нарушения углеводного обмена: внутри группы получавших длительную медикаментозную профилактику метформином не было выявлено статистически значимых различий при сопоставлении по исходному диагнозу НГН или НТГ (табл. 2).

Даже краткосрочная (только в период активного наблюдения, т. е. в течение трех лет) медикаментозная профилактика СД 2-го типа может влиять на результат, полученный через 10 лет наблюдения (табл. 3).

Эфффективность краткосрочной медикаментозной профилактики в долгосрочном периоде оказалась выше для НГН, чем для НТГ ( $p=0,012$ - для нормализации гликемии; $p=0,004$ - для конверсии в СД 2-го типа), что может быть связано с более высокой частотой назначения метформина, чем акарбозы.

\section{Нежелательные явления}

В ходе исследования не было зафиксировано непредвиденных нежелательных реакций (ННР), связанных с медикаментозной профилактикой СД 2-го типа. Учет нежелательных явлений, за исключением ННР, в рамках настоящего исследования в связи с особенностями его дизайна не был проведен.

\section{Ограничения исследования}

Ограничения исследования следуют из его дизайна: наблюдательный характер и использование только рутинных процедур не позволили ссормировать группы положительного и отрицательного контроля. То, что в течение 10 лет пациенты проходили диагностику и лечение по месту жительства, а не под контролем исследователей, не позволяет полностью исключить влияние внешних факторов на результаты исследования.

\section{ОБСУЖДЕНИЕ РЕЗУЛЬТАТОВ}

Вопросы приверженности к медикаментозной профилактике СД 2-го типа неотделимы от вопросов скрининга. 
Отсутствие мотивации для своевременного выявления ранних нарушений углеводного обмена в медицинском сообществе порождает отсутствие осведомленности, и, как следствие, отсутствие приверженности к медикаментозной терапии ранних нарушений углеводного обмена в сообществе пациентов.

Согласно стандартам медицинской помощи ADA 2019 [10, 11], применение метформина, ингибиторов $\alpha$-глюкозидазы, орлистата, агонистов ГПП-1 и тиазолидиндионов способствовало снижению заболеваемости СД 2-го типа, однако ни один из этих препаратов не включает предиабет в качестве зарегистрированного показания к применению. Опубликован протокол исследования SiMePred, которое должно оценить эфффективность ингибитора ДПП-4 ситаглиптина в профилактике СД 2-го типа, однако результаты данной работы пока недоступны [12]. Эксперты ADA считают метформин предпочтительной терапией для вторичной профилактики СД 2-го типа. Для остальных вышеперечисленных препаратов рекомендуют оценивать соотношение пользы и риска в каждом конкретном случае [10]. Между тем, в рекомендациях IDF от 2019 г. указана возможность назначения как метформина, так и ингибитора $\alpha$-глюкозидазы акарбозы с целью медикаментозной просилактики СД 2-го типа у лиц с начальными нарушениями углеводного обмена [13]. Следует отметить, что действующие в России инструкции по применению метформина и акарбозы содержат предиабет как показание к применению [14, 15].

В 2005 г. в Российской Федерации в рамках национального проекта «Здоровье» была принята целевая подпрограмма «Сахарный диабет» [16]. Ее целевыми индикаторами были выбраны продолжительность жизни больных сахарным диабетом и доля осложнений при сахарном диабете, т. е. упор был сделан на третичную профилактику.
В качестве программы третичной просилактики также можно рассматривать электронный государственный регистр сахарного диабета, но данных по его долговременной эффективности в качестве профилактического инструмента в настоящее время недостаточно.

В качестве первичной профилактики, на наш взгляд, следует рассматривать проведение диспансеризации населения [17], т. е. активное выявление факторов риска СД 2-го типа с последующим внедрением просилактических мер, направленных на уменьшение вклада модифицируемых факторов риска, а также своевременный скрининг не только в связи с диспансеризацией, но и в связи с наличием факторов риска. На практике требования по пробоподготовке не всегда выполняются, что приводит к гиподиагностике нарушений углеводного обмена. Согласно докладу экспертов ВОЗ (2006 г.), если при взятии образца крови немедленное отделение плазмы невозможно, образец цельной крови должен быть помещен в пробирку, содержащую ингибитор гликолиза (кодируется крышкой серого цвета), которую следует хранить во льду до выделения плазмы или проведения анализа. Этот цвет крышек утвержден Международной организацией стандартизации (ISO, 2000 г.) [18]

Вторичная профилактика СД 2-го типа включает два направления: наиболее раннее выявление заболевания и систему мер по замедлению конверсии начальных нарушений углеводного обмена в явный СД 2-го типа. Вторичная просилактика тесно связана с первичной, так как для пациентов, имеющих один или несколько факторов риска развития СД 2-го типа, охваченных рамками первичной профилактики, вторичная профилактика должна носить скрининговый характер. Федеральная программа вторичной профилактики СД 2-го типа, т. е. предотвращения прогрессии предиабета

Таблица 1. Результаты обследования пациентов, получавших и не получавших медикаментозную профилактику, в отдаленном периоде наблюдения

\begin{tabular}{|c|c|c|c|c|c|c|}
\hline \multirow{3}{*}{ Состояние углеводного обмена } & \multicolumn{4}{|c|}{ Число обследованных больных $n=115$} & \multirow{3}{*}{$\chi^{2}$} & \multirow{3}{*}{$p$} \\
\hline & \multicolumn{2}{|c|}{ Медикаментозная профилактика $n=30$} & \multicolumn{2}{|c|}{ Без медикаментозной профилактики $n=85$} & & \\
\hline & абс. & $\%$ & абс. & $\%$ & & \\
\hline Норма & 25 & 83,3 & 22 & 25,8 & 30,28 & $<0,05$ \\
\hline $\mathrm{HГH}$ & 1 & 3,3 & 13 & 15,2 & 3,0 & НД \\
\hline НTГ & 4 & 13,3 & 17 & 20,0 & 0,66 & НД \\
\hline СД 2-го типа & 0 & 0 & 33 & 38,8 & 16,3 & $<0,01$ \\
\hline
\end{tabular}

Примечание: НД - недостоверно.

Таблица 2. Отдаленные результаты ПтТГ пациентов группы длительной медикаментозной профилактики с учетом их исходного состояния углеводного обмена

\begin{tabular}{|c|c|c|c|c|}
\hline \multirow{2}{*}{$\begin{array}{c}\text { Результат обследования } \\
\text { через } 10 \text { лет }\end{array}$} & \multicolumn{2}{|c|}{ Исходное состояние пациентов группы длительной профилактики $(n=30)$} & \multirow{2}{*}{$\chi^{2}$} & \multirow{2}{*}{$p$} \\
\hline & $\mathrm{H} \Gamma \mathrm{H}$ & НТГ & & \\
\hline Норма & 21 & 4 & 0,250 & $>0,05$ \\
\hline $\mathrm{HГH}$ & 1 & 0 & 1 & $>0,05$ \\
\hline НТГ & 2 & 2 & 0,169 & $>0,05$ \\
\hline СД 2-го типа & 0 & 0 & - & - \\
\hline
\end{tabular}

Таблица 3. Результаты обследования пациентов группы без длительной медикаментозной профилактики с учетом их исходного состояния углеводного обмена

\begin{tabular}{|c|c|c|c|c|}
\hline \multirow{2}{*}{$\begin{array}{l}\text { Результат обследования через } \\
10 \text { лет }\end{array}$} & \multicolumn{2}{|c|}{$\begin{array}{c}\text { Исходное состояние пациентов группы без длительной } \\
\text { медикаментозной профилактики }(n=85)\end{array}$} & \multirow[t]{2}{*}{$\chi^{2}$ (с поправкой Йетса) } & \multirow[t]{2}{*}{$p$} \\
\hline & НГН & HTГ & & \\
\hline Норма & $17(20,0 \%)$ & $5(5,9 \%)$ & 6,4 & 0,012 \\
\hline НГН & $9(10,5 \%)$ & $4(4,8 \%)$ & 1,14 & 0,28 \\
\hline НTГ & $8(9,4 \%)$ & $9(10,5 \%)$ & 0,027 & 0,82 \\
\hline СД 2-го типа & $10(11,8 \%)$ & $23(27,1 \%)$ & 8,6 & 0,004 \\
\hline
\end{tabular}


в диабет, на сегодняшний день отсутствует. В связи с этим вторичная медикаментозная профилактика СД 2-го типа, продемонстрировавшая свою эффективность в отдаленном периоде как в нашем, так и в международных исследованиях [19, 20], может быть социально и экономически эффективным методом снижения бремени ранних нарушений углеводного обмена и СД 2-го типа.

\section{ВЫВОДЫ}

1. Отсутствие обязательного учета и диспансерного наблюдения пациентов с ранними нарушениями углеводного обмена приводит к недооценке клинического значения НТГ/
НГН, позднему выявлению СД 2-го типа и недостаточной приверженности пациентов к терапии. 2. Показано, что применение метформина позволило в условиях реальной клинической практики отсрочить развитие СД 2-го типа на длительное время. 3. Необходимо включать рекомендации по более активному выявлению НТГ/НГН и более раннему началу медикаментозной профилактики НТГ/НГН в алгоритмы и стандарты диагностики и лечения, используемые в рутинной практике. 4. При отсутствии противопоказаний всем лицам с начальными нарушениями углеводного обмена целесообразно назначение медикаментозной терапии метформином на неопределенно длительный срок.

\section{Литература}

1. Государственный регистр сахарного диабета Профессиональный Всероссийский ресурс по нозологиям диабета под эгидой Эндокринологического Научного Центра. Доступно по ссылке: http://diaregistry.ru/content/o-proekte. html\#content (проверено 28.05.18).

2. Дедов И. И., Шестакова М. В., редакторы. Алгоритмь специализированной медицинской помощи больным сахарным диабетом. 9-е изд. М., 2019.

3. Дедов И. И., Шестакова М. В., Галстян Г. Р. Распространенность сахарного диабета 2-го типа у взрослого населения России (исследование NATION). Сахарный диабет. 2016; 19 (2): 104-12.

4. Аметов А. С. Сахарный диабет 2-го типа. Проблемы и решения. M.: GEOTAR-Media, 2017; c. 125-44.

5. Barry E. Efficacy and effectiveness of screen and treat policies in prevention of type 2 diabetes: systematic review and meta-analysis of screening tests and interventions. BMJ. 2017 Jan 4; (356): i6538.

6. Аметов А. С., Кривошеева А. А. Профилактика развития сахарного диабета типа 2. Эндокринология. 2017; (4): 14-25.

7. Чазова И. Е., Мычка В. Б., Беленков Ю. Н. Основные результаты Российской программы АПРЕЛЬ (эфффективность применения акарбозы у пациентов с нарушенной толерантностью к глюкозе и артериальной гипертонией) Consilium medicum. 2005; (2): 18-22.

8. Madsen KS, Chi Y, Metzendorf MI, Richter B, Hemmingsen B. Metformin for prevention or delay of type 2 diabetes mellitus and its associated complications in persons at increased risk for the development of type 2 diabetes mellitus. Cochrane Database of Systematic Reviews. 2019; (12): CD008558

9. Демидова И. Ю., Боева В. В. Ранняя диагностика и лечение начальных стадий нарушений углеводного обмена. Вестник Российского государственного медицинского университета. 2013; (1): 9-13.

10. Diabetes Care. 2019; 42 (Suppl. 1): 29-33.

11. Аметов А. С., Кривошеева А. А. Перспективы ранней фармакологической интервенции на этапе предиабета.

\section{References}

1. State Diabetes Register. Professional All-Russian Resource on Diabetes Nosology under the auspices of Endocrinology Research Center. Available from: http://diaregistry.ru/content/oproekte.html\#content. Verified on May 28, 2018. Russian.

2. Dedov II, Shestakova MV, editors. Algorithms of Specialized Medical Care for Diabetes Mellitus Patients. 9th ed. Moscow, 2019. Russian

3. Dedov II, Shestakova MV, Galstyan GR. Prevalence of Type 2 Diabetes Mellitus in Adult Russian Population (NATION study). Diabetes Mellitus. 2016; 19 (2): 104-12. Russian.

4. Ametov AS. Saharnyj diabet 2-go tipa. Problemy i reshenija. M.: GEOTAR-Media, 2017; s. 125-144. Russian.

5. Barry E. Efficacy and effectiveness of screen and treat policies in
Эндокринология. 2018; 7 (3): 75-87.

12. Naidoo $P$, et al. Effect of Sitagliptin and Metformin on prediabetes progression to type 2 diabetes - a randomized, double-blind, double-arm, multicenter clinical trial: protocol for the sitagliptin and metformin in prediabetes (SiMePreD) study. JMIR Res Protoc. 2016; 5 (3): e145

13. International Diabetes Federation. Clinical Practice Recommendations for managing Type 2 Diabetes in Primary Care, 2017.

14. Государственный реестр лекарственных средств. Инструкция по применению лекарственного препарата для медицинского применения глюкофраж, изменение № 4 к П N014600/01140812 от 6.07.2016 г. С. 3. Дата переоформления: 04.04.2018 г.

15. Государственный реестр лекарственных средств. Инструкция по медицинскому применению препарата глюкобай. Регистрационный номер П N012033/01 от 24.06.2005 г. С. 2. Дата переоформления: 24.08.2017 г.

16. Дедов И. И., Шестакова М. В., редакторы. Результать реализации подпрограммы «Сахарный диабет» Федеральной целевой программы «Предупреждение и борьба с социально значимыми заболеваниями 2007-2012 годы». Сахарный диабет. 2013; (2): 2-48

17. Приказ Минздрава России от 3 декабря 2012 г. № 1006н. Доступно по ссылке: https://normativ.kontur.ru/document?mod uleld=1\&documentld=212999

18. Долгов В. В., Селиванова А. В. и др. Лабораторная диагностика нарушений обмена углеводов. Метаболический синдром. Сахарный диабет. М.-Тверь: Триада, 2006; 128 с.

19. Chiasson J-L, Josse RG, Gomis R, Hanefeld M, Karasik A, Laakso M, et al. Acarbose for prevention of type 2 diabetes mellitus: the STOP-NIDDM randomised trial. Lancet. 2002 Jun 15; 359 (9323): 2072-7.

20. Wenying $Y$, Lixiang $L$, Jinwu $Q$. The preventive effect of acarbose and metformin on the progression to diabetes mellitus in the IGT population: 3-year multicenter prospective study. Chin J Endocrin Metab. 2001; (17): 131-6.

prevention of type 2 diabetes: systematic review and meta-analysis of screening tests and interventions. BMJ. 2017 Jan 4; (356): i6538.

6. Ametov AS, Krivosheyeva AA. Prevention of type 2 diabetes mellitus. Endocrinology. 2017; (4): 14-25.

7. Chazova IE, Mychka VB, Belenkov YuN. Osnovnye rezul'taty Rossijskoj programmy "APREL" (jeffektivnost' primenenija akarbozy u pacientov s narushennoj tolerantnost'ju k gljukoze i arterial'noj gipertoniej). Consilium medicum. 2005; (2): 18-22. Russian.

8. Madsen KS, Chi Y, Metzendorf MI, Richter B, Hemmingsen B. Metformin for prevention or delay of type 2 diabetes mellitus and its associated complications in persons at increased risk for the development of type 2 diabetes mellitus. Cochrane Database of Systematic Reviews. 2019; (12): CD008558 
9. Demidova IYu, Boeva W. Early diagnosis and treatment of the initial stages of carbohydrate metabolism disorders. Bulletin of The Russian State Medical University. 2013; (1): 9-13.

10. Diabetes Care. 2019; 42 (Suppl. 1): 29-33.

11. Ametov AS, Krivosheyeva AA. Prospects of early pharmacological intervention at the stage of prediabetes. Endocrinology. 2018; 7 (3): 75-87.

12. Naidoo P, et al. Effect of Sitagliptin and Metformin on prediabetes progression to type 2 diabetes - a randomized, double-blind, double-arm, multicenter clinical trial: protocol for the sitagliptin and metformin in prediabetes (SiMePreD) study. JMIR Res Protoc. 2016; 5 (3): e145.

13. International Diabetes Federation. Clinical Practice Recommendations for managing Type 2 Diabetes in Primary Care, 2017.

14. Gosudarstvennyj reestr lekarstvennyh sredstv. Instrukcija po primeneniju lekarstvennogo preparata dlja medicinskogo primenenija gljukofazh, izmenenie \# 4 k P N014600/01-140812 ot 6.07.2016 g. S. 3. Data pereoformlenija: 04.04 .2018 g. Russian.

15. Gosudarstvennyj reestr lekarstvennyh sredstv. Instrukcija po medicinskomu primeneniju preparata gljukobaj. Registracionnyj nomer P N012033/01 ot 24.06.2005 g. S. 2. Data pereoformlenija: 24.08.2017 g. Russian.

16. Dedov II, Shestakova MV, redaktory. The results of the implementation of the "Diabetes mellitus" subprogram of the Federal target program "Prevention and Control of Socially Significant Diseases 2007-2012". Diabetes; 2013; (2): 2-48.

17. Prikaz Minzdrava Rossii ot 3 dekabrja 2012 g. \# 1006n. Available from: https://normativ.kontur.ru/document?moduleld=1\&docume $n t l d=212999$. Russian.

18. Dolgov W, Selivanova AV, i dr. Laboratornaja diagnostika narushenij obmena uglevodov. Metabolicheskij sindrom. Saharnyj diabet. M.-Tver': Triada, 2006; 128 s. Russian.

19. Chiasson J-L, Josse RG, Gomis R, Hanefeld M, Karasik A, Laakso M, et al. Acarbose for prevention of type 2 diabetes mellitus: the STOP-NIDDM randomised trial. Lancet. 2002 Jun 15; 359 (9323): 2072-7.

20. Wenying $Y$, Lixiang $L$, Jinwu $Q$. The preventive effect of acarbose and metformin on the progression to diabetes mellitus in the IGT population: 3-year multicenter prospective study. Chin J Endocrin Metab. 2001; (17): 131-6. 